\title{
Who Would Have Predicted Multisystem Inflammatory Syndrome in Children?
}

\author{
Daniel D. Reiff ${ }^{1}$ (I) $\cdot$ Randy Q. Cron ${ }^{1}$ (D)
}

Accepted: 14 December 2021 / Published online: 12 February 2022

(c) The Author(s), under exclusive licence to Springer Science+Business Media, LLC, part of Springer Nature 2022

\begin{abstract}
Purpose of Review Multisystem inflammatory disease in children (MIS-C) is a novel post-infectious phenomenon following coronavirus disease-19 (COVID-19). Herein, we present an in-depth review of the latest MIS-C literature related to clinical findings, pathophysiology, imaging and laboratory studies, treatment algorithms, and disease outcomes.

Recent Findings With its non-specific presentation of fever, gastrointestinal symptoms, cardiovascular injury and shock, systemic inflammation, and Kawasaki disease (KD)-like features, MIS-C can be a diagnostic challenge, overlapping with KD and active COVID-19 infection. However, common laboratory features, imaging findings, and historical clues can lead to accurate diagnosis and allow for appropriate treatment with a variety of immunomodulatory therapies, including intravenous immunoglobulin (IVIG). Aggressive treatment of MIS-C leads to good outcomes. Longitudinal studies continue to illuminate long-term cardiac sequelae and recovery.
\end{abstract}

Summary MIS-C presents with fever, KD features, gastrointestinal symptoms, cardiac inflammation, and shock. Early recognition and prompt institution of IVIG and glucocorticoids provide for rapid improvement.

Keywords COVID-19 $\cdot$ MIS-C $\cdot$ Inflammation $\cdot$ Pediatric rheumatology $\cdot$ Kawasaki disease $\cdot$ Cytokine

\section{Introduction}

Since its appearance in late 2019, the pandemic of severe acute respiratory syndrome coronavirus 2 (SARS-CoV-2), causing coronavirus disease 2019 (COVID-19), has infected over 200 million people worldwide and has caused over 4.5 million deaths. Ranging from asymptomatic to mild upper respiratory infectious symptoms to severe clotting abnormalities, shock, and acute respiratory distress syndrome, COVID-19 has had the most severe impact on the adult population, with the pediatric population relatively less affected during acute infection. However, early into the COVID-19 pandemic, a report arose out of the United Kingdom (UK) describing a cohort of 8 patients who

This article is part of the Topical Collection on Pediatric Rheumatology

Randy Q. Cron

randycron@uabmc.edu

1 Division of Rheumatology, Department of Pediatrics, Children's of Alabama, University of Alabama at Birmingham, 1600 7th, Ave. S, CPPN \#G10, Birmingham, AL 35233-1711, USA presented with hyperinflammatory shock, findings similar to Kawasaki disease (KD), gastrointestinal symptoms, and coronary artery changes, requiring significant cardiovascular support and immunomodulatory therapy [1•]. This was one of the first articles to report a presentation of the novel multisystem inflammatory syndrome in children (MIS-C), which has since affected tens of thousands across the globe. Other reports came from Europe describing a novel pandemic within a pandemic [2•]. This came to most everyone's surprise, although similar KD-like clusters have been reported previously in association with coronavirus infections, prompting some to postulate the KD should really be Kawasaki syndrome [3, 4]. Over the last year and a half, much has been learned about MIS-C, and herein, we review this influential and novel disease as it continues to affect children both in the United States (US) and abroad.

\section{Definitions of MIS-C}

Multiple central agencies have published case definitions and diagnostic criteria for MIS-C (Table 1). The United States Centers for Disease Control defined MIS-C as an individual 


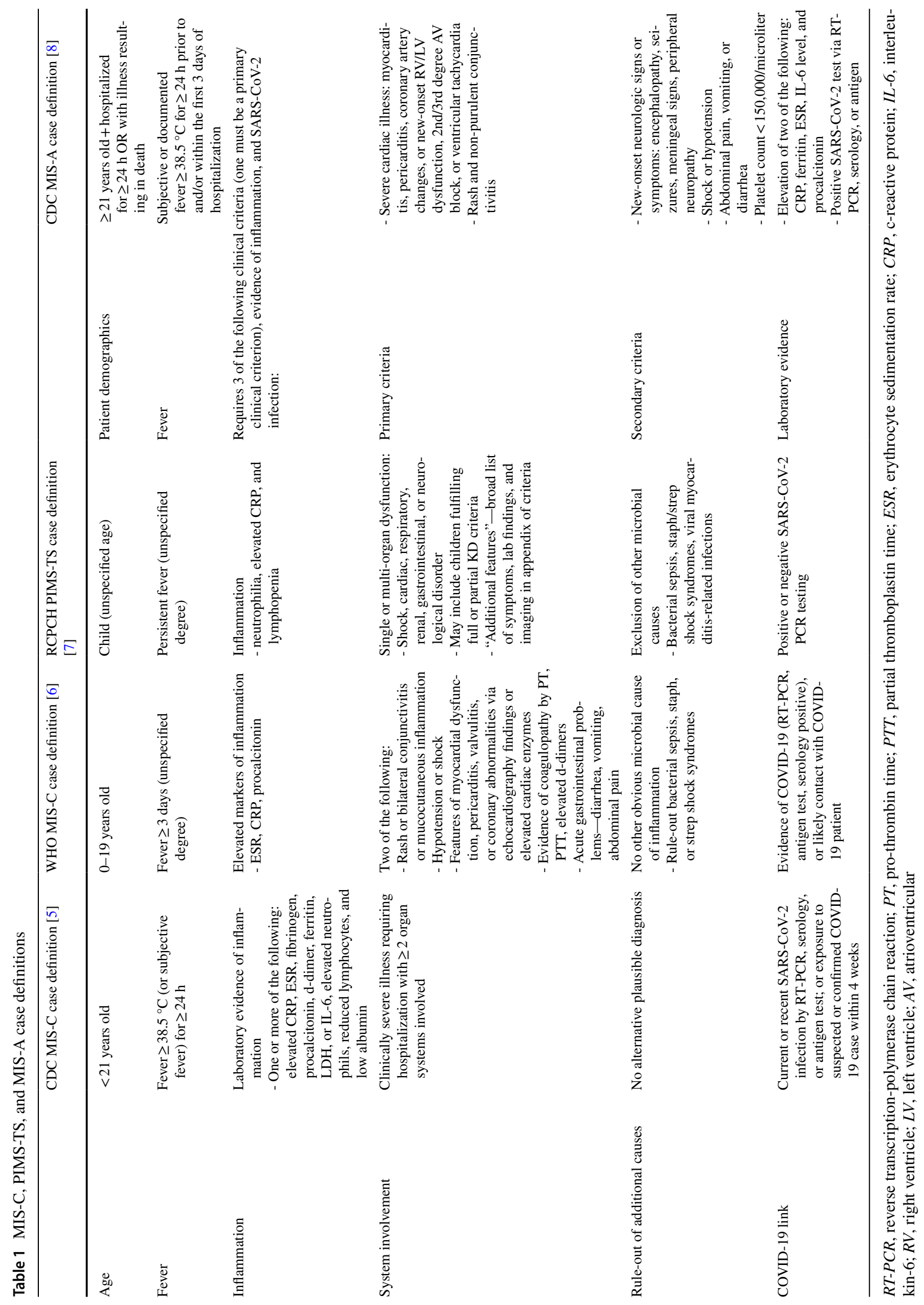


less than 21 years of age presenting with fever, elevated inflammatory markers, and severe illness with greater than two organ systems involved, and positive COVID-19 serology, SARS-CoV-2 antigen test, polymerase chain reaction (PCR), or exposure to COVID-19 within the 4 weeks prior to symptoms, and no alternative diagnoses [5]. The World Health Organization added their slightly varied definition, including patients $0-19$ years of age with fever for at least 3 days, elevated markers of inflammation, no other obvious source, evidence of COVID-19 via PCR, antigen testing, serologies, or likely sick contact, and two of the following: rash, conjunctivitis, or mucocutaneous inflammation; hypotensive shock; cardiac dysfunction; coagulopathy; or gastrointestinal symptoms [6]. In the UK, MIS-C is alternatively known as pediatric multisystem inflammatory syndrome temporally associated with SARS-CoV-2 (PIMS-TS) and is more broadly defined: a child presenting with fever, inflammation, positive or negative SARS-CoV-2 PCR, and organ system dysfunction with additional possible clinical, imaging, and laboratory features (such as abdominal pain, Kawasaki-like symptoms, gastrointestinal symptoms, cardiac dysfunction), excluding other microbial causes [7]. Regardless of the definition used, the common threads of a pediatric patient presenting with fever, inflammation, multiple organ system dysfunction, and severe illness are present in all criteria.

Starting in June 2020, reports arose of similar hyperinflammatory shock and severe illness in the adult population, leading to the defining of the multisystem inflammatory syndrome in adults (MIS-A) by the Centers for Disease Control. MIS-A is defined in patients greater than or equal to 21 years of age by similar criteria of severe cardiac illness, new-onset neurological symptoms, shock/hypotension, abdominal symptoms, thrombocytopenia, rash or conjunctivitis, evidence of systemic inflammation, and positive SARS-CoV-2 testing [8].

\section{Epidemiology and Demographics}

During the COVID-19 pandemic, children have been relatively spared in both infection rate, illness severity, and mortality. Since its onset, pediatric patients have accounted for $12.8 \%$ of total COVID-19 cases and $0.1 \%$ of total deaths, despite accounting for $22.3 \%$ of the total US population [9]. However, given that pediatric patients and younger children are more likely to be asymptomatic during their acute COVID-19 infection, it is likely that pediatric COVID-19 cases have been underestimated due to missed infections and undertesting since the appearance of this novel disease.

Comparatively, among the millions of pediatric patients diagnosed with active COVID-19, MIS-C seems to be a rare but serious manifestation. In the largest population study of 1733 MIS-C patients, the incidence was reported to be 2.1 cases per 100,000 children and, in line with the established case definitions and the post-infectious illness theory, peaks in MIS-C cases followed local COVID-19 outbreaks by $2-5$ weeks [10]. Incidence seems to differ by age, with an incidence of 2.3-2.9 cases per 100,000 children in 0-9 year olds, decreasing to $0.4-1.5$ per 100,000 in 15 to 20 -year-old patients [10].

When analyzing the six largest studied ( $>100$ patients) MIS-C cohorts of international and US children, the median age of the affected patient is 7-8.9 years of age, and data shows a slight male predominance of $54.7-67.8 \%$ of cases, similar to KD [10-12, 13•, 14]. MIS-C patients seem to be overwhelmingly healthy at baseline as $69.1-88.4 \%$ of patients did not have any prior medical conditions $[12,13 \bullet$, 15]. Consistent throughout most US MIS-C studies are broad racial and ethnic differences in prevalence and incidence of MIS-C. Black and Hispanic children are overrepresented in US MIS-C cohorts, making up $25-34.7 \%$ and $31-37.4 \%$ of patients diagnosed with MIS-C respectively, despite representing $13.4 \%$ and $18.5 \%$ of the total US population, per the most recent census data $[10,12,13 \bullet, 16]$. This discrepancy is likely multifactorial, as Black and Hispanic patients have also been affected by active COVID-19 infection at similar discordant rates. Racial and ethnic minority background and low socioeconomic status has been shown to increase the risk for SARS-CoV-2 infection in both the adult and pediatric population, and increased the risk for morbidity and death in adults [17, 18]. It is unclear what proportion of these racial and ethnic differences in MIS-C development is related to risk for COVID-19 infection and/or appropriate access to medical care; further study needs to be pursued to evaluate the reason for this discrepancy.

\section{Pathophysiology}

The pathophysiology of MIS-C is a much-debated topic in the literature, with no consensus yet identified. MIS-C is believed to be a post-infectious phenomenon, occurring a number of weeks after a primary COVID-19 infection. National geographic and temporal associations have been found between peaks of MIS-C cases and COVID-19 infection rates in the USA, with MIS-C peaks following peaks in COVID-19 infections by $2-5$ weeks $[10,13 \bullet, 19 \bullet]$. This post-infectious hypothesis is given further weight as the majority of MIS-C patients is positive for IgG antibodies against SARS-CoV-2 and has significantly lower SARSCoV-2 PCR cycle thresholds from nasopharyngeal viral testing as compared to active COVID-19 infection, indicating viral clearance $[11,12,15,19 \bullet, 20 \bullet \bullet]$.

Along with findings of a post-infectious pathogenesis, MIS-C is typified by a specific hyperinflammatory response 
and there have been many looking to profile this hyperinflammation with a multitude of biomarkers studied. From a cytokine and chemokine standpoint, high levels of interleukin (IL) $1 \beta$, IL-6, IL-8, IL-10, IL-17, interferon- $\gamma$ (IFN- $\gamma$ ), and CXCL-9 have been identified in MIS-C patients, with

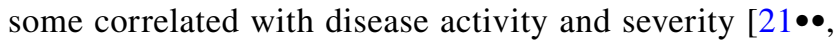
$22 \bullet \bullet, 23,24]$. Specifically, CXCL-9 (a marker of IFN- $\gamma$ ) levels have been shown to correlate with the level of inflammatory markers and cardiac dysfunction seen in MIS-C [25]. Studies have also shown activation of monocytes and neutrophils, decreased levels of circulating T-cells and natural killer cells, and lower total levels of B cells, highlighting the importance of certain immune cell lines in MIS-C disease activity and the recovery of these cell lines in disease recovery $[21 \bullet \bullet, 23,26]$.

Another mechanism of disease activity hypothesized is endothelial dysfunction leading to shock and cardiac injury and pro-coagulation leading to microvascular injury. MIS-C patients have been shown to have elevated levels of angiopoietin-2 (associated with vascular injury) and high angiopoietin-2/angiopoetin-1 ratios correlated with the level of shock severity and heart failure [27]. Additionally, secondary activation of auto-reactive antibodies arises in the MIS-C disease process, with some of these auto-antibodies targeted to antigens expressed in endothelial and cardiac tissues, as well as the gastrointestinal tract [23]. Along with this endothelial activation, thromboelastometry of MIS-C samples demonstrates hypercoagulability within the hyperinflammatory state [28].

\section{Presentation and Clinical Findings}

\section{Presenting Symptoms}

The presenting symptoms of MIS-C vary significantly in their severity and presence throughout the disease spectrum. Fever is the predominant symptom in MIS-C, usually persisting for 2-4 days prior to diagnosis, and as such is the mainstay of most diagnostic criteria $[11,12,15,19 \bullet]$. Gastrointestinal symptoms of nausea/vomiting, diarrhea, and abdominal pain are common complaints, present in 60-90\% of patients in the largest cohorts of patients [11, 12, 15, 19•]. Mucocutaneous and Kawasaki-like symptoms of conjunctivitis, rash, and mucosal changes are also frequent within MIS-C, appearing in $60-70 \%$ of all patients and seen in increased frequency in younger MIS-C patients [12, 19•]. Cardiovascular involvement has been reported in a significant proportion of MIS-C patients at $60-80 \%$ with tachycardia and hypotension among the most commonly reported symptoms [11, 12, 15, 19•]. Respiratory involvement has been reported variably and inconsistently in the literature, as its definition diverges widely from cough/congestion to respiratory failure requiring invasive mechanical ventilation. It is unclear as to the true respiratory involvement in MIS-C as respiratory failure and ventilatory needs can be due to primary disease activity, pulmonary edema from intensive volume resuscitation, and secondary to cardiovascular shock. Similar variable reporting is seen in neurologic system involvement, as definitions vary from headache and altered mental status to stroke, encephalitis, and demyelinating disorders. Neurologic symptoms have been reported in 11-40\% of MIS-C patients dependent on the definition employed [11, $12,15,19 \bullet]$. Other less common findings reported include lymphadenopathy, thrombosis, muscle aches and pains, and joint pain.

MIS-C symptoms are hardly specific and can involve almost any organ system with varying severity and timing. However, there have been important studies that have helped to identify different phenotypes under the MIS-C umbrella and to assist in improved diagnosis and treatment. Godfred-Cato et al. analyzed a 570 patient MIS-C cohort via latent class analysis and identified three distinct groupings of patients [29]. One group was likely active COVID-19 infection that was misclassified, but the other two groups separated MIS-C patients into two different subsets. The first MIS-C subset identified had very high frequency of cardiovascular and gastrointestinal involvement, with a significantly higher prevalence of abdominal pain, shock, myocarditis, inflammatory marker elevation, and SARSCoV-2 serology positivity. The second MIS-C subset was significantly younger with a higher prevalence of Kawasakilike mucocutaneous symptoms and rash, frequent coronary changes, and with less shock than the first MIS-C group [29]. Similar findings have been demonstrated by Geva et al., who identified features that cluster patients into a high likelihood of having MIS-C: previously healthy status at baseline, cardiovascular and mucocutaneous involvement, gastrointestinal symptoms, and high levels of inflammation and brain natriuretic peptide (BNP) [30].

\section{Laboratory Values and SARS-CoV-2 Testing}

Despite its wide array of clinical characteristics and symptomatology, MIS-C does have some consistent laboratory findings that are seen on presentation. White blood cell (WBC) count is generally normal to elevated with absolute lymphopenia, normal to elevated neutrophil counts, and high neutrophil/lymphocyte ratio, like COVID-19. Platelet count can be normal or depressed, with thrombocytopenia correlating with disease severity, and a post-inflammatory thrombocytosis can be seen, similar to that in $\operatorname{KD}[11,12,15,19 \bullet]$. Elevation in liver enzymes and creatinine can be seen in cases with hypotension, severe inflammation, and end-organ hypoperfusion. Inflammatory markers are quite elevated, with high levels of C-reactive protein (CRP), erythrocyte 
sedimentation rate (ESR), and ferritin seen in the majority of cases. Coagulation abnormalities are seen with elevation in d-dimer and fibrinogen, and lactate dehydrogenase (LDH) levels are elevated. During the disease course with ongoing vascular leak and third spacing, albumin and hemoglobin levels decrease and slowly normalize with treatment. Finally, those with significant cardiac involvement have elevated levels of troponin and BNP [11, 12, 15, 19•].

As previously discussed, a high percentage of MIS-C patients are positive for SARS-CoV-2 IgG antibodies against the nucleocapsid protein, indicating past infection with COVID-19. Cohorts vary widely in the percentage of MIS-C patients testing positive and range from 60-90\% positive for SARS-CoV-2 IgG antibodies [11, 12, 15, 19•]. However, the case definitions allow for PCR positivity to count toward the diagnostic criteria, and studies have shown that MIS-C patients can be simultaneously positive for both SARS-CoV-2 PCR and IgG in $30-40 \%$ of cases $[11,12,15$, 19•]. This dual positivity can create a diagnostic dilemma for providers, muddying the picture between MIS-C and active COVID-19. However, it is likely that these dual-positive patients are not actively infectious. As mentioned previously, MIS-C patients have higher PCR cycle thresholds (indicating lower viral RNA levels) than active COVID-19 patients and additional studies have shown that PCR can remain positive in nasopharyngeal samples for a significant amount of time after viral clearance tested in culture [20••, 31].

\section{Cardiac Findings}

In patients with cardiovascular involvement, echocardiography, cardiac magnetic resonance imaging (MRI), and electrocardiography demonstrate findings of myocardial dysfunction, coronary dilatation/aneurysms, and conduction abnormalities. EKG is performed in a proportion of MIS-C patients and studies vary widely in the prevalence of abnormalities. Abnormal EKGs have been reported in 35.3-67\% of patients on presentation with low QRS amplitudes, transient $T$ wave inversion, and arrhythmias [14, 32]. Studies have found arrhythmias in upwards of $8-21 \%$ of patients with AV block seen in 2-20\% [12, 14, 33, 34]. Echocardiography in MIS-C patients shows reduced left ventricular systolic function and ejection fraction in $30-40 \%$ of patients and can show valvular regurgitation and pericardial effusion $[12,13 \bullet, 14,35,36]$. Similar to KD, MIS-C has been associated with coronary artery aneurysms and dilatation; echocardiography has shown dilatation and/or aneurysm in $13-28 \%$ of patients $[12,14,35]$. Cardiac MRI is less frequently performed in acute MIS-C and is most often used to monitor the recovery of patients after hospitalization. However, cardiac MRI in small hospitalized cohorts has found T2 hyperintensity, pericardial effusions, myocardial edema, and late gadolinium enhancement without evidence of focal necrosis or fibrosis $[14,37]$.

\section{Non-cardiac Imaging}

Non-cardiac imaging in MIS-C can show a wide variety of findings that point toward systemic inflammation of multiple organ systems. In children with significant abdominal symptoms, common findings on abdominal computed tomography (CT) were ascites, hepatomegaly, and localized ileitis or diffuse bowel wall edema [38, 39, 40•]. In the setting of predominant gastrointestinal symptoms, those with localized ileitis may be at increased risk for an incorrect diagnosis of appendicitis, especially if imaged only with appendix ultrasound [41]. Chest CT and radiography in MIS-C patients can demonstrate residual ground-glass opacities, pleural effusion, consolidation, and peribronchial thickening [39, $40 \bullet, 42]$. In patients with neurologic abnormalities, brain imaging can be helpful to differentiate between reversible or fulminant neurologic findings, as MRI has demonstrated a wide variety of findings including ischemic or hemorrhagic stroke, white matter hyperintensities, corpus callosum changes, and demyelination [43•].

\section{Differentiation of MIS-C from KD and Acute COVID-19}

\section{MIS-C and KD}

MIS-C and KD are very similar in the acute presentation, and the similarity of these two disease processes guided much of the initial treatment algorithms for MIS-C. However, there are some key differences that differentiate the two disease processes, both in their clinical presentation and pathogenesis. Patients with MIS-C are more likely to present with cardiac, gastrointestinal, coagulopathic, and neurologic symptomology than patients with KD and more often require intensive care $[44,45]$. KD patients are generally younger at disease onset with a lower prevalence of cytopenias and a lower degree of hyperferritinemia than those with MIS-C $[44,45]$. These differences in the clinical presentation can possibly be explained by subtle differences in the inflammatory profiles seen with these two disease processes. Both KD and MIS-C show high levels of inflammatory cytokines and chemokines, including IFN- $\gamma$, IL-18, and IL- $1 \alpha$, but differ in the expression of CXCL9, with MIS-C patients expressing higher levels [25, 46].

\section{MIS-C and Active COVID-19}

Throughout the pandemic and especially in locations with constant community spread of SARS-CoV-2, peaks of 
MIS-C and active COVID-19 infections have significant overlap, creating a diagnostic dilemma for healthcare providers. With somewhat non-specific lab findings and clinical presentations, much work has been done to differential active COVID-19 infection and post-infectious MIS-C, as they require different workup and treatment. Patients with active COVID-19 infection are more likely than MIS-C patients to have underlying medical conditions and to be of a racial or ethnic minority [12, 47-49]. On presentation, fever, gastrointestinal involvement, cardiac involvement, and KDlike symptoms were more common in MIS-C patients, where COVID-19 infected patients were more likely to suffer from primary respiratory symptoms [12, 47-49]. Patients with MIS-C have higher neutrophil counts, higher neutrophil to lymphocyte ratios, higher levels of inflammation (CRP and ESR), lower levels of LDH, and lower platelet counts than those with COVID-19 [12, 47-49]. Therapies for MIS-C and COVID-19 are similar, but not identical.

\section{Treatment}

There are no universally accepted treatment algorithms for MIS-C to date, but many institutions and medical societies have published individual guidelines that have changed and adapted throughout the pandemic. A general treatment algorithm can be found in Table 2 .

\section{Supportive Cares}

Initial treatment of MIS-C is focused on immediately treating life-threatening sequelae of the disease process including cardiac dysfunction, respiratory failures, and end-organ injury. The range of illness severity seen with MIS-C is broad, ranging from supportive care measures in mild cases to invasive mechanical ventilator support and extracorporeal membrane oxygenation (ECMO) in severe cases. In the largest cohorts of MIS-C patients, vasoactive/inotropic medications were required in $14.7-45 \%$ of cases, invasive ventilatory measures were required in $15.3-23.5 \%$ of patients, and ECMO was required in $0.3-4 \%[11,12,13 \bullet, 14]$. Many children with MIS-C present severely ill, often requiring intensive care. Fortunately, immunomodulatory therapies are typically rapidly effective.

\section{Immunomodulatory Therapies}

Since the initial reports of MIS-C cases, the mainstays of immunomodulatory treatment have been intravenous immunoglobulin (IVIG) and corticosteroids, given MIS-C's similarity to KD and its proposed hyperinflammatory pathogenesis. IVIG is well known to prevent coronary aneurysm in KD and is therefore the initial immunomodulatory medication of choice in the most proposed treatment algorithms at $2 \mathrm{~g}$ per kilogram ( $\mathrm{g} / \mathrm{kg}$ ) dosing [50-53]. The next line medication in most MIS-C treatment algorithms is glucocorticoids, with dosing and timing of administration varying widely. Treatment algorithms generally break up steroid dosing into two groups: lower doses (1-2 mg/kg/day) used for those patients without severe cardiac manifestation or shock and higher doses (10-30 mg/kg/day) reserved for those with severe features, coronary changes, and/or shock [51-53]. In the largest cohorts of MIS-C patients, IVIG was used in $77-89 \%$ of patients with steroid use varying more widely at $28-69.4 \%$ $[11,12,13 \bullet, 14,19 \bullet]$. Early in the pandemic, corticosteroids were used more sparingly and in sequential order after IVIG, but more recent data has shown improved outcomes with early steroid use with or without IVIG. Son et al. reported that IVIG plus steroid treatment was associated with a lower risk of cardiovascular complications and adjunctive therapy requirement as compared to IVIG alone, and Ouldali et al. showed that IVIG plus methylprednisolone versus IVIG alone was associated with lower rates of treatment failure, lower hemodynamic support requirements, and decreased length of ICU stay $[54 \bullet, 55 \bullet]$. Smaller studies have shown that corticosteroid use alone can even be superior to IVIG use as a first-line medication, with faster normalization of left ventricular function, fever curve, and shorter ICU stay [56]. This is particularly relevant to resource-poor settings.

Many different adjunctive therapies and immunomodulatory medications have been used with varying efficacy in MIS-C. Treatment algorithms generally recommend biologic agents as third-line medication options after initial treatment with IVIG and steroids. Anakinra is an IL-1 receptor antagonist that has been previously been shown to be effective with limited side effects in KD patients [57]. Multiple studies have shown anakinra to be similarly effective in MIS-C, especially when it comes to cardiac function, after failure or poor response to IVIG and steroid treatment [58-60]. In two large cohorts, anakinra was used in 24/186 and $8 / 183$ patients to good effect $[11,13 \bullet]$. Infliximab is a tumor necrosis factor inhibitor that has long been used in IVIG-refractory KD and has been demonstrated to be similarly effective in refractory cases of MIS-C [61, 62]. Many other therapies have been referenced as possible nextline medications in the literature, including tocilizumab and plasmapheresis.

\section{Anticoagulation and Thrombosis Risk}

MIS-C has been shown to have pro-thrombotic features with early elevations in d-dimers and fibrinogen, late elevation in platelet count, and evidence of hypercoagulability on thromboelastometry [28]. These findings and the similarity of MIS-C to KD have led to the generally 
Table 2 MIS-C treatment algorithm

Hospitalized patient with MIS-C [51-53]

Patient hemodynamically stable without severe features

First-line treatment

IVIG $2 \mathrm{~g} / \mathrm{kg}$

Monitor for improvement in fever curve, inflammatory markers

Second-line treatment for refractory disease

Addition of methylprednisolone $1-2 \mathrm{mg} / \mathrm{kg} /$ day

Consider alternative second-line agent (anakinra, infliximab, etc.)

Additional medications

Initiate anti-platelet therapy with aspirin $3-5 \mathrm{mg} / \mathrm{kg}$ daily (max. $81 \mathrm{mg}$ / day)

If on steroids and aspirin, initiate gastric prophylactic medication
Patient with hypotensive shock, respiratory failure, severe neurologic involvement

First-line treatment

IVIG $2 \mathrm{~g} / \mathrm{kg}$ + methylprednisolone $1-2 \mathrm{mg} / \mathrm{kg} /$ day

If life or organ threatening disease, consider methylprednisolone $10-30 \mathrm{mg} / \mathrm{kg} /$ day

Second-line treatment for refractory disease

Intensify steroid regimen to methylprednisolone $10-30 \mathrm{mg} / \mathrm{kg} / \mathrm{day}$

Addition of high-dose anakinra or alternative second-line agent

Initiate anticoagulation vs anti-platelet therapy; hold anti-platelet therapy for significant thrombocytopenia

If on high-dose steroids \pm aspirin, initiate gastric prophylactic medication uniform addition of anti-platelet agents to MIS-C treatment algorithms. In most cases, a low dose of 3-5 mg/ $/ \mathrm{kg} /$ day of aspirin (up to $81 \mathrm{mg}$ daily) is recommended in patients with Kawasaki-like findings, coronary changes, and thrombocytosis [51-53]. If patients require both steroids and aspirin treatment, the generally accepted practice is to add gastric prophylaxis medication as well to prevent ulceration. Individual treatment algorithms have differing opinions on the use of anticoagulation in lieu of aspirin in the setting of severe disease with abnormal cardiac function, ventilatory requirements, and ICU admission. Treatment options include low molecular weight heparin, enoxaparin, and/or discussion with hematologists on an individual case basis [51-53].

\section{Prognosis and Outcomes}

Overall, MIS-C patients overwhelmingly do well with appropriate therapy and a vast majority recover from their illness, regardless of severity. However, there are those patients who do not recover and death has been reported in $0.3-2 \%$ of MIS-C cases, higher than the mortality of pediatric active COVID-19 infection, reported to be $<0.1 \%$ of pediatric cases $[11,12,13 \bullet, 14,63]$. Increased risk of death from MIS-C is associated with patients of racial and ethnic minorities, ages 16-20 years and is seen in those with severe cardiac involvement and/or existing underlying medical conditions [64]. In those patients who survive, there is undoubtedly a long road to recovery, especially in those who require significant cardiorespiratory support with vasopressors, ventilation, and ECMO. Unfortunately, there is a lack of longitudinal studies focusing specifically on patients who require intensive interventions, but they are incorporated in other types of longitudinal studies.

Systemic inflammation has been shown to rapidly improve with appropriate treatment, including two studies of 46 and 45 MIS-C patients showing normalization of C-reactive protein, platelet count, liver enzyme levels, albumin levels, and d-dimer levels in the vast majority by $4-6$ months, and in some by as early as 6 weeks [ 65 , 66]. Cardiac function abnormalities have been shown to improve in a similar manner. In a study of 50 MIS-C patients, LV systolic dysfunction was seen at diagnosis in $52 \%$ of patients, with only 1 patient having persistent dysfunction on echocardiography at 2-week follow-up and complete resolution at 8 weeks [67•]. Additional studies have utilized cardiac MRI at follow up for more specific analysis of cardiac function. In a study of 20 patients referred for follow-up cardiac MRI, 50\% had LV ejection fraction $<55 \%$ during acute MIS-C, but all had complete normalization of all cardiac abnormalities within 2-6 weeks after discharge [68]. Another study of 19 consecutive MIS-C patients with LV ejection fraction $<55 \%$ showed no persistent cardiac changes at a median follow-up of 99 days post-hospitalization [69]. Finally, a large source of concern for medical providers is the development and persistence of coronary artery dilatation and aneurysm formation in MIS-C patients. Coronary artery dilatation $(\mathrm{z}>2)$ has been reported in 9-24.1\% of MIS-C cases [11, 12, 13•, 14]. However, in multiple follow-up studies, coronary changes have been shown to resolve with appropriate treatment-one study reported 7 out of $45(15.6 \%)$ patients with coronary changes on admission, all resolved within 1-4 weeks [66], and another study demonstrated coronary changes in 12 out of 50 (24\%) patients that resolved by 8 weeks 
to 6 months [67•]. Overall, those MIS-C patients that are appropriately treated and recover from the initial disease course have promising longitudinal outcomes, with continued research ongoing.

\section{Conclusion}

MIS-C is a novel, post-infectious manifestation of SARS$\mathrm{CoV}-2$ with characteristics similar to KD and typified by intense inflammation throughout the body, affecting most organ systems. Although clinical symptoms and laboratory findings can be somewhat non-specific, unique patterns have been identified to aid clinicians in appropriate diagnosis and differentiation from similar disease processes. When treated appropriately with immunomodulatory therapy, early longitudinal data has shown relatively optimistic outcomes for recovery of overall health and cardiac dysfunction. However, current cohorts of MIS-C patients will need to be followed further, to fully grasp the long-term effects of this condition. Further study is also needed into the pathophysiology of MIS-C to develop more targeted therapies and in anticipation of post-infectious inflammatory conditions that may be of public health concern in the future.

Acknowledgements The authors thank the tireless and timely contributions from physician-scientists across the globe detailing this novel disease, multisystem inflammatory syndrome in children, MIS-C.

Author contribution This review article was produced on the invitation. Dr. Reiff performed the literature search with additional articles supplied by Dr. Cron. The initial draft was written by Dr. Reiff with editing and revisions by Dr. Cron.

Availability of data and material All source data and materials comply with field standards and will be made available to interested parties upon request.

Code Availability Not applicable.

\section{Declarations}

Ethics Approval Not applicable.

Consent to Participate Not applicable.

Conflict of Interest Dr. Reiff has no conflicts of interest to disclose. Dr. Cron discloses the following: work for SOBI on the advisory board for MAS, as a paid speaker/moderator for MEDSCAPE/WebMD, and investigator initiating a clinical trial of anakinra to treat MAS; consulting for Novartis and Sironax; and work for Pfizer on a clinical trial for MAS adjudication committee chair.

\section{References}

Papers of particular interest, published recently, have been highlighted as:

- Of importance

$\bullet$ Of major importance

1.• Riphagen S, Gomez X, Gonzalez-Martinez C, Wilkinson N, Theocharis P. Hyperinflammatory shock in children during COVID19 pandemic. Lancet. 2020;395(10237):1607-8. https://doi.org/ 10.1016/S0140-6736(20)31094-1. (These reports are some of the earliest descriptions of the novel KD-like hyperinflammatory condition later termed, MIS-C.)

2. Verdoni L, Mazza A, Gervasoni A, et al. An outbreak of severe Kawasaki-like disease at the Italian epicentre of the SARS-CoV-2 epidemic: an observational cohort study. Lancet. 2020;395(10239):1771-8. https://doi.org/10.1016/S0140$6736(20) 31103-X$. (These reports are some of the earliest descriptions of the novel KD-like hyperinflammatory condition later termed, MIS-C.)

3. Esper F, Shapiro ED, Weibel C, Ferguson D, Landry ML, Kahn JS. Association between a novel human coronavirus and Kawasaki disease. J Infect Dis. 2005;191(4):499-502. https://doi.org/ 10.1086/428291.

4. Ravelli A, Martini A. Kawasaki disease or Kawasaki syndrome? Ann Rheum Dis. 2020;79(8):993-5. https://doi.org/10.1136/ annrheumdis-2020-218110.

5. Centers for Disease Control and Prevention. Multisystem Inflammatory Syndrome in Children (MIS-C) Associated with coronavirus disease 2019 (COVID-19). May 14th 2020. (Accessed August 9, 2021). https://emergency.cdc.gov/han/2020/han00432.asp

6. World Health Organization. Multisystem inflammatory syndrome in children and adolescents with COVID-19. May 15th 2020. (Accessed August 9, 2021). https://www.who.int/news$\mathrm{room} / \mathrm{commentaries/detail/multisystem-inflammatory-syndr}$ ome-in-children-and-adolescents-with-covid-19

7. Royal College of Paediatrics and Child Health. Paediatric multisystem inflammatory syndrome temporally associated with COVID-19 (PIMS) - guidance for clinicians. May 12020. (Accessed August 9, 2021). https://www.rcpch.ac.uk/resources/ paediatric-multisystem-inflammatory-syndrome-temporallyassociated-covid-19-pims-guidance

8. Centers for Disease Control and Prevention. Multisystem inflammatory syndrome in adults (MIS-A) case definition information for healthcare providers. May 11 2021. (Accessed August 10, 2021). https://www.cdc.gov/mis/mis-a/hcp.html

9. Centers for Disease Control and Prevention. Demographic trends of COVID-19 cases and deaths in the US reported to CDC. August 9 2021. (Accessed August 10, 2021). https://covid. cdc.gov/covid-data-tracker/?CDC_AA_refVal=https $\% 3 \mathrm{~A} \% 2 \mathrm{~F} \%$ 2Fwww.cdc.gov\%2Fcoronavirus\%2F2019-ncov\%2Fcases-updat es\%2Fcases-in-us.html\#demographics

10. Belay ED, Abrams J, Oster ME, et al. Trends in geographic and temporal distribution of US children with multisystem inflammatory syndrome during the COVID-19 pandemic. JAMA Pediatr. 2021;175(8):837-45. https://doi.org/10.1001/jamapediatrics. 2021.0630 .

11. Bautista-Rodriguez C, Sanchez-de-Toledo J, Clark BC, et al. Multisystem inflammatory syndrome in children: an international survey. Pediatrics. 2021;147(2): e2020024554. https:// doi.org/10.1542/peds.2020-024554.

12. Feldstein LR, Tenforde MW, Friedman KG, et al. Characteristics and outcomes of US children and adolescents with multisystem 
inflammatory syndrome in children (MIS-C) compared with severe acute COVID-19. JAMA. 2021;325(11):1074-87. https:// doi.org/10.1001/jama.2021.2091.

13. Feldstein LR, Rose EB, Horwitz SM, et al. Multisystem inflammatory syndrome in U.S. children and adolescents. $\mathrm{N}$ Engl J Med. 2020;383(4):334-46. https://doi.org/10.1056/ NEJMoa2021680. (This is one of the first reports to detail the demographic, clinical, and laboratory features of a relatively large cohort $(n=186)$ of children suffering hospitalized MIS-C across the United States. Therapeutics and outcomes were also reported.)

14. Valverde I, Singh Y, Sanchez-de-Toledo J, et al. Acute cardiovascular manifestations in 286 children with multisystem inflammatory syndrome associated with COVID-19 infection in Europe. Circulation. 2021;143(1):21-32. https://doi.org/10. 1161/CIRCULATIONAHA.120.050065.

15. Antúnez-Montes OY, Escamilla MI, Figueroa-Uribe AF, et al. COVID-19 and multisystem inflammatory syndrome in Latin American Children: a multinational study. Pediatr Infect Dis J. 2021;40(1):e1-6. https://doi.org/10.1097/INF.0000000000 002949 .

16. United States Census Bureau. Quickfacts. July 12019. (Accessed August 10, 2021). https://www.census.gov/quick facts/fact/table/US/PST045219

17. Goyal MK, Simpson JN, Boyle MD, et al. Racial and/or ethnic and socioeconomic disparities of SARS-CoV-2 infection among children. Pediatrics. 2020;146(4): e2020009951. https://doi.org/10.1542/peds.2020-009951

18. Javalkar K, Robson VK, Gaffney L, et al. Socioeconomic and racial and/or ethnic disparities in multisystem inflammatory syndrome. Pediatrics. 2021;147(5): e2020039933. https://doi. org/10.1542/peds.2020-039933.

19. Dufort EM, Koumans EH, Chow EJ, et al. Multisystem inflammatory syndrome in children in New York State. N Engl J Med. 2020;383(4):347-58. https://doi.org/10.1056/NEJMo a2021756. (This is one of the first reports to detail the demographic, clinical, and laboratory features of a relatively large cohort $(n=191)$ of children suffering hospitalized MIS-C in New York state. Therapeutics and outcomes were also reported.)

20.• Diorio C, Henrickson SE, Vella LA, et al. Multisystem inflammatory syndrome in children and COVID-19 are distinct presentations of SARS-CoV-2. J Clin Invest. 2020;130(11):5967-75. https://doi.org/10.1172/JCI140970. (These 3 studies detailed distinct clinical presentations between pediatric COVID-19 and MIS-C early during the pandemic. Descriptions of the hematologic and immunologic phenotypes detailed the various cytokine profiles and white blood cell subsets relatively unique to children with MIS-C.)

21.• Carter MJ, Fish M, Jennings A, et al. Peripheral immunophenotypes in children with multisystem inflammatory syndrome associated with SARS-CoV-2 infection. Nat Med. 2020;26(11):1701-7. https://doi.org/10.1038/s41591-020-10546. (These 3 studies detailed distinct clinical presentations between pediatric COVID-19 and MIS-C early during the pandemic. Descriptions of the hematologic and immunologic phenotypes detailed the various cytokine profiles and white blood cell subsets relatively unique to children with MIS-C.)

22.• Lee PY, Day-Lewis M, Henderson LA, et al. Distinct clinical and immunological features of SARS-CoV-2-induced multisystem inflammatory syndrome in children. J Clin Invest. 2020;130(11):5942-50. https://doi.org/10.1172/JCI141113. (These 3 studies detailed distinct clinical presentations between pediatric COVID-19 and MIS-C early during the pandemic. Descriptions of the hematologic and immunologic phenotypes detailed the various cytokine profiles and white blood cell subsets relatively unique to children with MIS-C.)

23. Gruber CN, Patel RS, Trachtman R, et al. Mapping systemic inflammation and antibody responses in multisystem inflammatory syndrome in children (MIS-C). Cell. 2020;183(4):982995.e14. https://doi.org/10.1016/j.cell.2020.09.034. (This study reported on autoantibody profiles (e.g. anti-La) detected in children with MIS-C.)

24. Peart Akindele N, Kouo T, Karaba AH, et al. Distinct cytokine and chemokine dysregulation in hospitalized children with acute coronavirus disease 2019 and multisystem inflammatory syndrome with similar levels of nasopharyngeal severe acute respiratory syndrome coronavirus 2 shedding. J Infect Dis. 2021;224(4):606-15. https://doi.org/10.1093/infdis/jiab285.

25. Rodriguez-Smith JJ, Verweyen EL, Clay GM, et al. Inflammatory biomarkers in COVID-19-associated multisystem inflammatory syndrome in children, Kawasaki disease, and macrophage activation syndrome: a cohort study. Lancet Rheumatol. 2021;3(8):e574-84. https://doi.org/10.1016/S2665-9913(21) 00139-9.

26. Okarska-Napierała M, Mańdziuk J, Feleszko W, et al. Recurrent assessment of lymphocyte subsets in 32 patients with multisystem inflammatory syndrome in children (MIS-C). Pediatr Allergy Immunol. 2021;32(8):1857-65. https://doi.org/10.1111/ pai.13611.

27. Borgel D, Chocron R, Grimaud M, et al. Endothelial dysfunction as a component of severe acute respiratory syndrome coronavirus 2-related multisystem inflammatory syndrome in children with shock. Crit Care Med. 2021;49(11):e1151-6. https://doi. org/10.1097/CCM.0000000000005093.

28. Al-Ghafry M, Vagrecha A, Malik M, et al. Multisystem inflammatory syndrome in children (MIS-C) and the prothrombotic state: coagulation profiles and rotational thromboelastometry in a MIS-C cohort. J Thromb Haemost. 2021;19(7):1764-70. https://doi.org/10.1111/jth.15340.

29. Godfred-Cato S, Bryant B, Leung J, et al. COVID-19-associated multisystem inflammatory syndrome in children - United States, March-July. MMWR Morb Mortal Wkly Rep. 2020;69(32):107480. https://doi.org/10.15585/mmwr.mm6932e2.

30. Geva A, Patel MM, Newhams MM, et al. Data-driven clustering identifies features distinguishing multisystem inflammatory syndrome from acute COVID-19 in children and adolescents. EClinicalMedicine. 2021;40: 101112. https://doi.org/10.1016/j. eclinm.2021.101112.

31. Kim MC, Cui C, Shin KR, et al. Duration of culturable SARSCoV-2 in hospitalized patients with Covid-19. N Engl J Med. 2021;384(7):671-3. https://doi.org/10.1056/NEJMc202704.

32. Regan W, O'Byrne L, Stewart K, et al. Electrocardiographic changes in children with multisystem inflammation associated with COVID-19: associated with coronavirus disease 2019. J Pediatr. 2021;234:27-32.e2. https://doi.org/10.1016/j.jpeds.2020. 12.033 .

33. Choi NH, Fremed M, Starc T, et al. MIS-C and cardiac conduction abnormalities. Pediatrics. 2020;146(6): e2020009738. https://doi.org/10.1542/peds.2020-009738.

34. Dionne A, Mah DY, Son MBF, et al. Atrioventricular block in children with multisystem inflammatory syndrome. Pediatrics. 2020;146(5): e2020009704. https://doi.org/10.1542/peds. 2020-009704.

35. Haghighi Aski B, Manafi Anari A, Abolhasan Choobdar F, Zareh Mahmoudabadi R, Sakhaei M. Cardiac abnormalities due to multisystem inflammatory syndrome temporally associated with Covid-19 among children: a systematic review and metaanalysis. Int J Cardiol Heart Vasc. 2021;33: 100764. https://doi. org/10.1016/j.ijcha.2021.100764. 
36. Kobayashi R, Dionne A, Ferraro A, et al. Detailed assessment of left ventricular function in multisystem inflammatory syndrome in children, using strain analysis. CJC Open. 2021;3(7):880-7. https://doi.org/10.1016/j.cjco.2021.02.012.

37. Blondiaux E, Parisot P, Redheuil A, et al. Cardiac MRI in children with multisystem inflammatory syndrome associated with COVID-19. Radiology. 2020;297(3):E283-8. https://doi.org/10. 1148/radiol.2020202288.

38. Morparia K, Park MJ, Kalyanaraman M, McQueen D, Bergel M, Phatak T. Abdominal imaging findings in critically ill children with multisystem inflammatory syndrome associated with COVID-19. Pediatr Infect Dis J. 2021;40(2):e82-3. https://doi. org/10.1097/INF.0000000000002967.

39. Fenlon Iii EP, Chen S, Ruzal-Shapiro CB, Jaramillo D, Maddocks ABR. Extracardiac imaging findings in COVID-19-associated multisystem inflammatory syndrome in children. Pediatr Radiol. 2021;51(5):831-9. https://doi.org/10.1007/ s00247-020-04929-1.

40. Caro-Domínguez P, Navallas M, Riaza-Martin L, et al. Imaging findings of multisystem inflammatory syndrome in children associated with COVID-19. Pediatr Radiol. 2021;51(9):160820. https://doi.org/10.1007/s00247-021-05065-0. (Among a multi-center cohort of 37 children with MIS-C (81\% requiring intensive care), chest, cardiac, and abdominal imaging revealed ground glass opacities of the lungs, impaired cardiac function, and terminal ileum thickening as common findings.)

41. Azılı MN, Güney D, Oztorun CI, et al. 2021 Determination of factors to distinguish MIS-C from acute appendicitis in children with acute abdominal pain [published online ahead of print, Jul 23]. Eur J Pediatr Surg. 2021; https://doi.org/10.1055/s-00411732320

42. Rostad BS, Shah JH, Rostad CA, et al. Chest radiograph features of multisystem inflammatory syndrome in children (MIS-C) compared to pediatric COVID-19. Pediatr Radiol. 2021;51(2):231-8. https://doi.org/10.1007/s00247-020-04921-9.

43. LaRovere KL, Riggs BJ, Poussaint TY, et al. Neurologic involvement in children and adolescents hospitalized in the United States for COVID-19 or multisystem inflammatory syndrome. JAMA Neurol. 2021;78(5):536-47. https://doi. org/10.1001/jamaneurol.2021.0504. (This large $(\mathbf{n = 1 , 6 9 5 )}$ pediatric COVID-19 and MIS-C case series reports $22 \%$ of children with documented neurologic disorders, including sever encephalopathy, stroke, and central nervous system demyelination.)

44. Chen MR, Kuo HC, Lee YJ, et al. Phenotype, susceptibility, autoimmunity, and immunotherapy between Kawasaki disease and coronavirus disease-19 associated multisystem inflammatory syndrome in children. Front Immunol. 2021;12:632890. https:// doi.org/10.3389/fimmu.2021.632890.

45. OtarYener G, PaçKısaarslan A, Ulu K, et al. Differences and similarities of multisystem inflammatory syndrome in children, Kawasaki disease and macrophage activating syndrome due to systemic juvenile idiopathic arthritis: a comparative study. Rheumatol Int. 2021;7(1):11. https://doi.org/10.1007/ s00296-021-04980-7.

46. Esteve-Sole A, Anton J, Pino-Ramirez RM, et al. Similarities and differences between the immunopathogenesis of COVID19-related pediatric multisystem inflammatory syndrome and Kawasaki disease. J Clin Invest. 2021;131(6): e144554. https:// doi.org/10.1172/JCI144554.

47. Zhao Y, Yin L, Patel J, Tang L, Huang Y. The inflammatory markers of multisystem inflammatory syndrome in children (MIS-C) and adolescents associated with COVID-19: a metaanalysis. J Med Virol. 2021;93(7):4358-69. https://doi.org/10. 1002/jmv.26951.
48. Reiff DD, Mannion ML, Samuy N, Scalici P, Cron RQ. Distinguishing active pediatric COVID-19 pneumonia from MIS-C. Pediatr Rheumatol Online J. 2021;19(1):21. https://doi.org/10. 1186/s12969-021-00508-2.

49. Tripathi S, Gist KM, Bjornstad EC, et al. Coronavirus disease 2019-associated PICU admissions: a report from the Society of Critical Care Medicine Discovery Network Viral Infection and Respiratory Illness Universal Study Registry. Pediatr Crit Care Med. 2021;22(7):603-15. https://doi.org/10.1097/PCC.00000 00000002760 .

50. Oates-Whitehead RM, Baumer JH, Haines L, et al. Intravenous immunoglobulin for the treatment of Kawasaki disease in children. Cochrane Database Syst Rev. 2003;2003(4):CD004000. https://doi.org/10.1002/14651858.CD004000.

51. Henderson LA, Canna SW, Friedman KG, et al. American College of Rheumatology Clinical Guidance for Multisystem Inflammatory Syndrome in Children Associated With SARSCoV-2 and Hyperinflammation in Pediatric COVID-19: Version 2. Arthritis Rheumatol. 2021;73(4):e13-29. https://doi.org/10. 1002/art.41616.

52. Schlapbach LJ, Andre MC, Grazioli S, et al. Best practice recommendations for the diagnosis and management of children with pediatric inflammatory multisystem syndrome temporally associated with SARS-CoV-2 (PIMS-TS; Multisystem Inflammatory Syndrome in Children, MIS-C) in Switzerland. Front Pediatr. 2021;9:667507. https://doi.org/10.3389/fped.2021.667507.

53. Harwood R, Allin B, Jones CE, et al. A national consensus management pathway for paediatric inflammatory multisystem syndrome temporally associated with COVID-19 (PIMS-TS): results of a national Delphi process. Lancet Child Adolesc Health. 2021;5(2):133-41. https://doi.org/10.1016/S2352-4642(20) 30304-7.

54. Son MBF, Murray N, Friedman K, et al. Multisystem inflammatory syndrome in children - initial therapy and outcomes. N Engl J Med. 2021;385(1):23-34. https://doi.org/10.1056/NEJMo a2102605. (One of two large retrospective case series $(\mathbf{n}=\mathbf{5 1 8})$ revealed improved outcomes of children with MIS-C who were treated with glucocortiocoids in addition to IVIG.)

55. Ouldali N, Toubiana J, Antona D, et al. Association of intravenous immunoglobulins plus methylprednisolone vs immunoglobulins alone with course of fever in multisystem inflammatory syndrome in children. JAMA. 2021;325(9):855-64. https:// doi.org/10.1001/jama.2021.0694. (One of two large retrospective case series $(n=111)$ revealed improved outcomes of children with MIS-C who were treated with glucocortiocoids in addition to IVIG.)

56. Vukomanovic V, Krasic S, Prijic S, et al. Recent experience: corticosteroids as a first-line therapy in children with multisystem inflammatory syndrome and COVID-19-related myocardial damage. Pediatr Infect Dis J. 2021;40(11):e390-4. https://doi. org/10.1097/INF.0000000000003260.

57. Kone-Paut I, Cimaz R, Herberg J, et al. The use of interleukin 1 receptor antagonist (anakinra) in Kawasaki disease: a retrospective cases series. Autoimmun Rev. 2018;17(8):768-74. https:// doi.org/10.1016/j.autrev.2018.01.024.

58. Mastrolia MV, Marrani E, Calabri GB, et al. Fast recovery of cardiac function in PIMS-TS patients early using intravenous anti-IL-1 treatment. Crit Care. 2021;25(1):131. https://doi.org/ 10.1186/s13054-021-03548-y.

59. Fouriki A, Fougère Y, De Camaret $\mathrm{C}$, et al. Case report: case series of children with multisystem inflammatory syndrome following SARS-CoV-2 infection in Switzerland. Front Pediatr. 2021;8:594127. https://doi.org/10.3389/fped.2020.594127.

60. Della Paolera S, Valencic E, Piscianz E, et al. Case report: use of Anakinra in multisystem inflammatory syndrome during 
COVID-19 pandemic. Front Pediatr. 2021;8:624248. https:// doi.org/10.3389/fped.2020.624248.

61. Burns JC, Best BM, Mejias A, et al. Infliximab treatment of intravenous immunoglobulin-resistant Kawasaki disease. J Pediatr. 2008;153(6):833-8. https://doi.org/10.1016/j.jpeds.2008.06. 011.

62. Abdel-Haq N, Asmar BI, Deza Leon MP, et al. SARS-CoV2-associated multisystem inflammatory syndrome in children: clinical manifestations and the role of infliximab treatment. Eur J Pediatr. 2021;180(5):1581-91. https://doi.org/10.1007/ s00431-021-03935-1.

63. Leidman E, Duca LM, Omura JD, Proia K, Stephens JW, Sauber-Schatz EK. COVID-19 Trends Among Persons Aged 0-24 Years-United States, March 1-December 12, 2020. MMWR Morb Mortal Wkly Rep. 2021;70(3):88-94. https://doi.org/10. 15585/mmwr.mm7003e1.

64. Bowen A, Miller AD, Zambrano LD, et al. Demographic and clinical factors associated with death among persons $<21$ years old with multisystem inflammatory syndrome in children-United States, February 2020-March 2021. Open Forum Infect Dis. 2021;8(8):ofab388. https://doi.org/10.1093/ofid/ofab388.

65. Penner J, Abdel-Mannan O, Grant K, et al. 6-month multidisciplinary follow-up and outcomes of patients with paediatric inflammatory multisystem syndrome (PIMS-TS) at a UK tertiary paediatric hospital: a retrospective cohort study. Lancet Child Adolesc Health. 2021;5(7):473-82. https://doi.org/10.1016/ S2352-4642(21)00138-3.

66. Farooqi KM, Chan A, Weller RJ, et al. Longitudinal outcomes for multisystem inflammatory syndrome in children. Pediatrics.
2021;148(2): e2021051155. https://doi.org/10.1542/peds. 2021-051155.

67. Capone CA, Subramony A, Sweberg T, et al. Characteristics, cardiac involvement, and outcomes of multisystem inflammatory syndrome of childhood associated with severe acute respiratory syndrome coronavirus 2 Infection. J Pediatr. 2020;224:141-5. https://doi.org/10.1016/j.jpeds.2020.06.044. (This is one of the first long-term (up to 9 months post hospital discharge) follow up studies of children with MIS-C $(n=45)$ revealing, that despite critical illness, most inflammatory and cardiac manifestations resolved rapidly.)

68. Bermejo IA, Bautista-Rodriguez C, Fraisse A, et al. Short-term sequelae of multisystem inflammatory syndrome in children assessed by CMR. JACC Cardiovasc Imaging. 2021;14(8):16667. https://doi.org/10.1016/j.jcmg.2021.01.035.

69. Bartoszek M, Małek ŁA, Barczuk-Falęcka M, Brzewski M. 2021 Cardiac magnetic resonance follow-up of children after pediatric inflammatory multisystem syndrome temporally associated with SARS-CoV-2 with initial cardiac involvement [published online ahead of print, $2021 \mathrm{Jul}$ 29]. J Magn Reson Imaging.https://doi. org/10.1002/jmri.27870. https://doi.org/10.1002/jmri.27870

Publisher's note Springer Nature remains neutral with regard to jurisdictional claims in published maps and institutional affiliations. 